[Article]

\title{
自组装半导体碳纳米管薄膜的光电特性
}

\author{
赵青鋔 ${ }^{1}$ 刘 昒 ${ }^{1,2}$ 魏 楠 ${ }^{1}$ 王 胜 ${ }^{1,}{ }^{*}$ \\ ('北京大学电子学系, 纳米器件物理与化学教育部重点实验室, 北京 100871; \\ 北京大学前沿交叉学科研究院, 北京 100871)
}

\begin{abstract}
摘要：采用自组装的方法制备 $99 \%$ 高纯度半导体碳纳米管平行阵列条带, 以金属钯和钪为非对称接触电极制 备碳纳米管(CNT)薄膜晶体管(TFTs)器件. 主要研究不同沟道长度碳纳米管薄膜晶体管器件的电输运特性和红 外光电响应特性, 分析了其中的载流子输运和光生载流子分离的物理机制. 我们发现薄膜晶体管器件的电学性 能和光电性能依赖于器件沟道长度 $(L)$ 和碳纳米管的平均长度 $\left(L_{\mathrm{CNT}}\right)$. 当沟道长度小于碳纳米管的平均长度时, 器件开关比最低; 当沟道长度超过碳纳米管平均长度时, 随着沟道长度的增加, 器件开关比增加, 光电流减小. 相关研究结果为高纯碳纳米管薄膜晶体管器件在红外光探测器方面的进一步应用提供参考依据.
\end{abstract}

关键词：碳纳米管；自组装；非对称接触；光电响应；红外；沟道长度；薄膜晶体管

中图分类号: 0649

\section{Photoelectric Characteristics of Self-Assembled Semiconducting Carbon Nanotube Thin Films}

\author{
ZHAO Qing-Liang ${ }^{1} \quad$ LIU Yang ${ }^{1,2} \quad$ WEI Nan ${ }^{1} \quad$ WANG Sheng ${ }^{1, *}$ \\ ('Key Laboratory for the Physics and Chemistry of Nanodevices, Department of Electronics, Peking University, \\ Beijing 100871, P. R. China; ${ }^{2}$ Academy for Advanced Interdisciplinary Studies, Peking University, Beijing 100871, P. R. China)
}

\begin{abstract}
We used the self-assembly method to form high purity (99\%) semiconducting carbon nanotube (CNT) aligned arrays. Thin-film transistors (TFTs) were fabricated with asymmetric Pd and Sc electrodes. We studied the electronic transport characteristics and infrared photoelectronic properties of the TFTs with different channel lengths. The physical mechanism of carrier transport and the dissociation of photoexcited carries are also discussed. We found that the electronic and photoelectronic properties of the TFTs were dependent on the channel length and the average length of the CNTs. The on/off ratio of the device was the lowest when the channel length of the device $(L)$ was less than the average length of the CNTs $\left(L_{\mathrm{CNT}}\right)$, and it increased with increasing $L$ when $L$ was larger than $L_{\text {сNт. }}$ In addition, the short circuit current of the device also decreased. These results provide an effective reference for further infrared detector applications based on high-purity semiconducting carbon nanotube TFTs.
\end{abstract}

Key Words: Carbon nanotube; Self-assembly; Asymmetric contact; Photoelectric response; Infrared; Channel length; Thin film transistor

\section{1 引 言}

碳纳米管(CNTs)自 1991 年被发现以来, 在纳
米电子和光电应用领域以其优异的性能和广阔的 应用潜力得到了广泛的关注. 在电学方面, 半导体

Received: February 26, 2014; Revised: May 6, 2014; Published on Web: May 9, 2014.

"Corresponding author. Email: shengwang@pku.edu.cn; Tel: +86-13671286710.

The project was supported by the National Key Basic Research Program of China (973) (2011CB933002, 2011CB933001) and National Natural

Science Foundation of China (61370009, 61271051, 61321001).

国家重点基础研究发展规划项目(973) (2011CB933002, 2011CB933001)和国家自然科学基金(61370009,61271051,61321001)资助

(C) Editorial office of Acta Physico-Chimica Sinica 
型单壁碳纳米管具有高达 $10^{5} \mathrm{~cm}^{2} \cdot \mathrm{V}^{-1} \cdot \mathrm{s}^{-1}$ 的载流子 迁移率 ${ }^{2}$ 和超过 $1 \mu \mathrm{m}$ 的电子平均自由程. ${ }^{3}$ 单根半导 体单壁碳纳米管作为沟道材料的场效应晶体管 (FET), 其性能指标已经在多方面超过传统硅基器 件. 此外, 碳纳米管还具有良好的化学稳定性和机 械延展性, 具有很好的构建柔性电子器件、 ${ }^{4}$ 全碳电 路的潜力. ${ }^{5,6}$ 在光电特性方面, 碳纳米管与传统光电 材料如化合物半导体、有机物半导体相比也具有优 异的光吸收和光响应性能. 碳纳米管是一种多子 带、直接带隙的半导体, 其带隙可调, 并与直径大致 成反比关系, ${ }^{7}$ 因此碳纳米管薄膜具有从紫外到红外 的宽谱光吸收特性. 碳纳米管的吸收系数很高, 已 报道碳管薄膜样品在近红外到中红外区间的光吸 收系数在 $10^{4}-10^{5} \mathrm{~cm}^{-1}$ 之间, 较传统红外材料高出 约一个量级. 作为一种小尺度的纳米材料, 碳纳米 管具有很好的光电集成潜力, 在保持较高探测性能 的同时, 单一像素器件能够达到亚微米尺度.

最早的碳纳米管场效应器件是由碳纳米管和 铂 $(\mathrm{Pt})$ 金属形成肖特基接触制备而成, 但肖特基结 的存在限制了器件的性能, ${ }^{10}$ 对于光电器件应用也 会限制最佳光电流和光电压的获得. 斯坦福大学的 Dai 研究组 ${ }^{11}$ 于 2003 年首次在碳管上采用高功函数 的钯 $(\mathrm{Pd})$ 金属作为源漏电极, 实现了 $p$ 型欧姆接触 的场效应晶体管, 器件的接触电阻接近理论的量子 极限. 我们研究组 ${ }^{12,13}$ 利用低功函数的钪 $(\mathrm{Sc})$ 和钎 $(\mathrm{Y})$ 金属实现了很好的碳管 $n$ 型欧姆接触. 利用 $\mathrm{Pd}$ 和 $\mathrm{Sc} / \mathrm{Y}$ 金属电极可以构建非对称接触的碳纳米管二 极管, ${ }^{14}$ 无需化学掺杂即可形成类似 $p-n$ 结的内建电 场, 可以高效地实现光生载流子的分离和收集. 基 于非对称接触碳管二极管, 经过引入 “虚电极”对可 以构成级联碳管二极管结构, 利用级联结构的光伏 倍增效应, ${ }^{15}$ 碳纳米管平行阵列作为沟道材料的光 探测器件可以得到更高的信噪比和探测度, 多级级 联探测器的室温红外探测率可以接近一般的商用 红外探测器水平. ${ }^{16}$

先前的基于单根半导体碳纳米管二极管器件 的电学和光电性能的研究显示出很好的应用潜 力. ${ }^{14,15}$ 但由于单根碳纳米管材料尺度的限制, 难以 满足应用所需材料的大规模制备和均匀分布要求. 碳纳米管薄膜材料在发挥单根碳管优异性能的同 时, 也可以在二维尺度上拓展碳管各方面的应用, 如电子器件的沟道材料 ${ }^{4,17-19}$ 和太阳能电池..$^{20}$ 目前 常用的薄膜制备方法主要有化学气相沉积法和溶
液沉积法. 虽然化学气相沉积直接生长可得到高质 量的碳纳米管薄膜, 但这种方式得到的碳管薄膜中 半导体性碳纳米管和金属性碳纳米管混合在一起, 通常直接生长的碳纳米管中金属管和半导体管的 比例为 $1: 2$, 金属管的存在会使器件具有较低的开关 比, 而且不利于半导体碳管中的光激发载流子的分 离. 如果不能有效去除金属管, 基于这种薄膜的器 件应用受到较大限制. 一般的溶液沉积法可以用密 度梯度超速离心法提纯后的碳纳米管做原料, 经过 自然沉积得到随机网络状分布的高纯度半导体碳 纳米管薄膜, 其中的碳管是无序分布状态, 并且碳 管的密度大面积均匀性较难控制. ${ }^{21}$ 在溶液沉积法 的诸多方式中, 蒸发自组装方法在排列的定向性和 大面积可控性上具有很大优势. 22 先前的结果显示 出用该方法得到的碳管薄膜材料制备的薄膜场效 应器件表现出了较好的均一性和电输运性能, ${ }^{23}$ 而 且由于碳纳米管的光吸收和光发射具有明显的偏 振依赖关系, ${ }^{24}$ 对于碳纳米管薄膜在光电器件方面 的应用来说, 需要定向排列的碳纳米管薄膜以确保 器件的光学特性控制.

本文采用密度梯度超速离心法提纯的 $99 \%$ 半 导体单壁碳纳米管作为材料, 利用蒸发自组装的方 法实现了碳纳米管大面积的近似平行排列的条带, 在此基础上批量制备了非对称接触薄膜晶体管器 件, 主要研究器件沟道长度和碳纳米管的平均长度 对器件的电学及光电性质的影响规律, 以及薄膜晶 体管沟道中光生载流子的分离特性.

\section{2 实验部分}

\section{1 碳纳米管阵列条带材料的制备与表征}

制备碳纳米管阵列的原材料是经过预先提纯 的固态 $99 \%$ 半导体碳纳米管 (美国 NanoIntegris 公 司). 首先将碳纳米管加入浓度为 $1 \%$ 的十二烷基硫 酸钠(SDS)溶液中, SDS 购于 Sigma 公司, 纯度大于 99\%. 用宁波新芝生物科技股份有限公司生产的 JY92-2D 型超声波细胞破碎机, 以 $200 \mathrm{~W}$ 功率超声 $1 \mathrm{~h}$, 以使碳管充分分散. 将超声处理过后的碳纳米 管的 SDS 溶液进行超速离心, 离心机为日本日立公 司 CS150GX II, 在 $104000 \mathrm{G}$ 的加速度下离心 30 min. 离心后取上层清液, 得到分散较好的碳纳米管 溶液, 将这些溶液进一步稀释 10 倍后, 将表面清洗 干净的 $500 \mathrm{~nm}$ 厚氧化硅的硅片垂直插入溶液中, 放 入真空干燥器, 在 0.4 个大气压下、减震环境下排列 
$48 \mathrm{~h}$, 得到大面积均一的定向排列的碳纳米管条带. 碳纳米管条带的扫描电子显微镜(SEM) 照片如图 1 (a)所示, 碳管条带的宽度约为 $18 \mu \mathrm{m}$, 条带中碳管大 致呈平行排列, 条带的宽度和周期大小可以通过碳 管的浓度和气压条件进行控制. 所用的经分离提纯 后的单根碳管的长度平均约为 $1 \mu \mathrm{m}$, 薄膜条带中碳 纳米管的管径分布可以由拉曼光谱表征得到. 图 1 $(b, c)$ 为碳管薄膜的拉曼光谱, 从图 1(b, c) 中可以看 到碳管样品明显的呼吸模(RBM)和 $G$ 模, 同时可以 看到较小的 $D$ 峰, 利用简单的 RBM 峰和直径关系 $\omega=248 / d$ (其中 $\omega$ 是 RBM 峰的位置, $d$ 是碳管以 $\mathrm{nm}$ 为单位时的直径大小), 可以得到碳管直径的分布范 围为 1.2-1.7 nm. ${ }^{25}$ 图 1(d) 为 99\%高纯度碳管薄膜的 光吸收谱, 吸收谱是采用美国 Perkin Elmer 公司的 Lambda 950 型紫外-可见分光光度计进行测量的. 在 1100 和 $1800 \mathrm{~nm}$ 附近显示出强的吸收峰, 它们可 以分别对应半导体碳纳米管 $\mathrm{S}_{22}$ 和 $\mathrm{S}_{11}$ 的吸收峰, 吸 收谱的长波截止波长在 $2500 \mathrm{~nm}$ 左右, 说明这种高 纯碳管薄膜在可见光到近红外波段均有显著光吸 收, 同时吸收谱的波长范围也与拉曼光谱给出的碳 管的直径分布基本一致. 吸收谱中未见明显的金属
性碳纳米管 $\mathrm{M}_{11}$ 吸收峰, 证明材料中半导体管有较 高的纯度. ${ }^{20}$

\section{2 碳纳米管阵列器件的制备}

主要采用电子束光刻(EBL)方式对前面获得的 $\mathrm{Si} / \mathrm{SiO}_{2}$ 祄底上的碳纳米管条带进行电极加工, 所用 的 EBL 是加载在美国 FEI 公司 XL 30 SFEG 型号的 SEM 上的德国 Raith 公司电子束图形发生器附件来 完成的. 用电子束蒸发镀膜的方式蒸镀金属电极, 所用仪器为美国 Kurt J. Lesker公司的电子束蒸发镀 膜仪. 镀膜之后随即通过美国 Cambridge 公司原子 层沉积(ALD)方式生长 $12 \mathrm{~nm}$ 的氧化铪进行保护, 随后进行剥离. 采用美国 Trion 公司的电感耦合等离 子体刻蚀仪(ICP)刻蚀掉器件四周的碳纳米管薄膜, 以保证器件之间的独立性. 最后用德国 Micro Chem 公司的电子束光刻胶聚甲基丙烯酸甲酯(PMMA), 浓度为 $6 \%$, 对器件进行封装, 以增加器件在大气中 测量的稳定性. 典型器件的结构如图 2(a) 所示, 以 $\mathrm{Si} / \mathrm{SiO}_{2}$ 做底栅, 源极和漏极金属分别为钛/钯 (宽度 $0.5 \mu \mathrm{m}$, 厚度 $0.5 \mathrm{~nm} / 60 \mathrm{~nm}$ ) 和钪(宽度 $0.5 \mu \mathrm{m}$, 厚度 $70 \mathrm{~nm})$.

\section{3 器件电学和光电性质的测量}
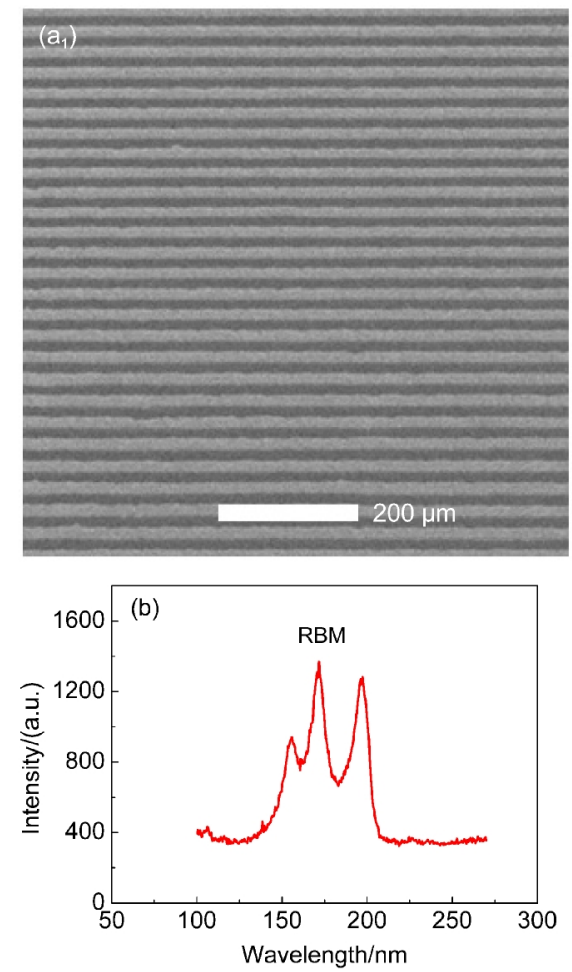
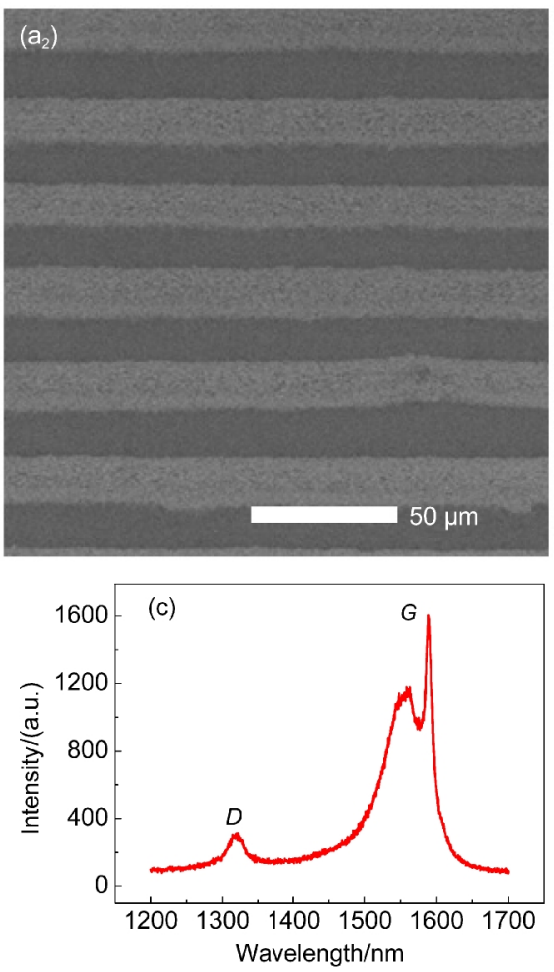
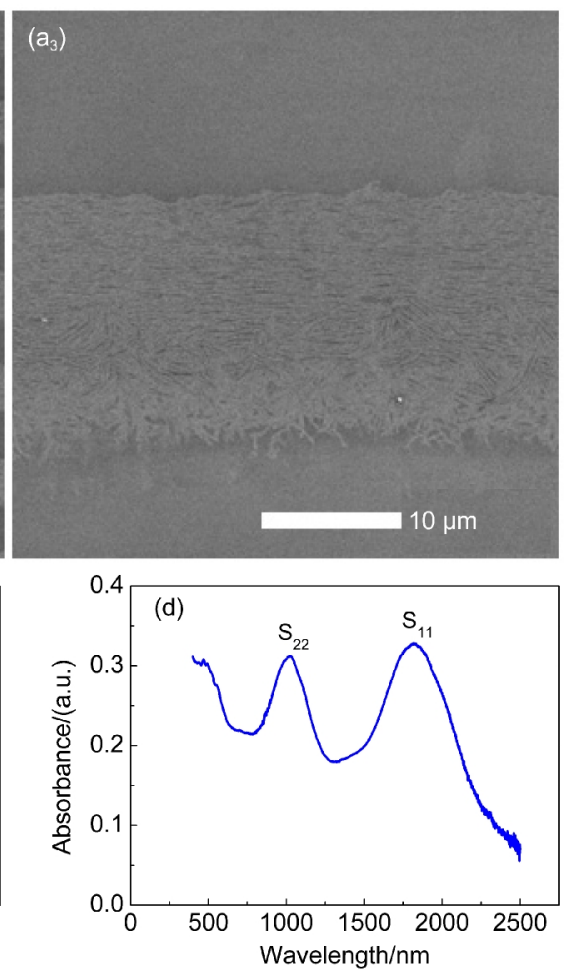

图 1 高纯半导体碳纳米管平行阵列薄膜的表征

Fig.1 Characterization of the high purity semiconducting nanotube aligned arrays

$\left(\mathrm{a}_{1}-\mathrm{a}_{3}\right)$ SEM image of the carbon nanotube film; (b) radial breathing mode (RBM), (c) $D$ band and $G$ band of Raman spectrum of the carbon nanotube film; (d) visible-near-infrared absorption spectra of carbon nanotube film on glass substrate 
器件的电学输运特性测量是在常温、大气环境 下进行的, 所用仪器为美国 Keitheley4200 SCS 半导 体分析仪以及探针台. 光电测量是在美国 Horiba JY 公司 HR 800 显微拉曼光学系统上通过自制的探针 台完成的, 用 Keithley4200 SCS 半导体分析仪进行 光电响应的测量. 所用激光波长为 785 和 $633 \mathrm{~nm}$.

\section{3 结果与讨论}

\section{1 器件的电学特性表征}

图 2(a)为碳纳米管薄膜条带器件结构示意图. $\mathrm{Si} / \mathrm{SiO}_{2}$ 为祄底的同时也作为底栅控制碳管薄膜晶 体管的开关状态, $\mathrm{Pd}$ 和 $\mathrm{Sc}$ 金属作为源漏电极与碳纳 米管形成非对称接触. 由于碳纳米管薄膜中的碳管 为准定向排列, 碳管的平均长度约为 $1 \mu \mathrm{m}$, 对于不 同沟道长度器件的输运机制不同, 在沟道比较短的 时候(小于 $1 \mu \mathrm{m}$ ), 沟道里大部分碳管以直接输运为 主, 即通过直接搭接在源漏两端电极, 当沟道比较
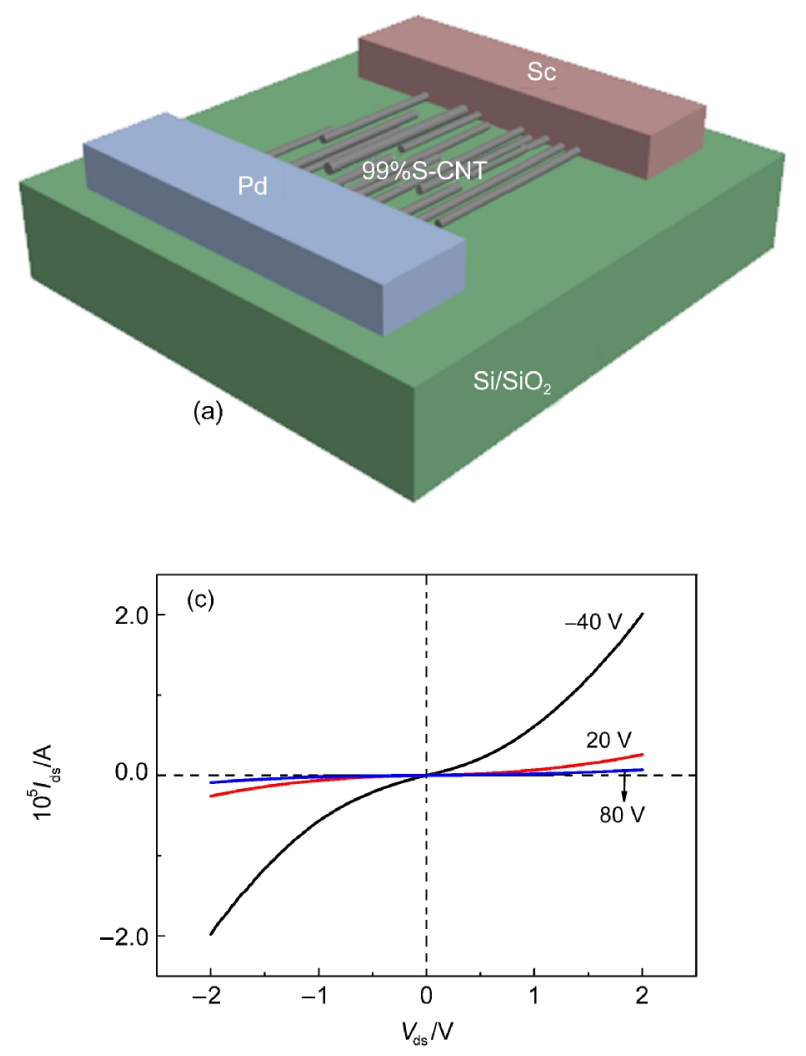

长的时候(大于 $1 \mu \mathrm{m})$, 沟道中的碳管无法直接连接 两端源漏电极, 主要以碳管相互搭接的方式连接, 即以渗透输运机制为主. 为了比较不同沟道长度器 件的输运特性, 我们首先研究了沟道长度对器件电 学特性的影响. 图 2(b) 显示了不同沟道长度(分别为 $0.5,1,2,5 \mu \mathrm{m})$ 器件的转移特性曲线 $\left(I_{\mathrm{ds}}-V_{\mathrm{gs}}\right.$ (ds: 源 漏极, gs: 栅源极)). 器件的沟道中碳管条带宽度均 约为 $18 \mu \mathrm{m}$, 栅压扫描范围为 $-80-80 \mathrm{~V}$, 漏极 $(\mathrm{Pd})$ 偏压为 $1 \mathrm{~V}$, 源极 $(\mathrm{Sc})$ 接地. 碳管薄膜晶体管器件的 转移特性呈现出典型的 $p$ 型特征. 对于不同的沟道 长度器件, 其阈值电压不同, 对于沟道较长 $(2,5 \mu \mathrm{m})$ 器件, 当其栅压 $\left(V_{\mathrm{gs}}\right)$ 为 $60 \mathrm{~V}$ 左右为关态, 对于沟道较 短器件 $(0.5,1 \mu \mathrm{m})$, 其关态电压为 20-40 V 之间, 阈 值电压的变化主要可能是来自不同沟道长度器件 的电输运机制. 随着沟道长度的增大, 器件的开关 比增大, 开态电流减小. 当沟道长度为 $0.5 \mu \mathrm{m}$ 时, 器 件具有最大的开态电导, 由于平均碳管长度约为 1
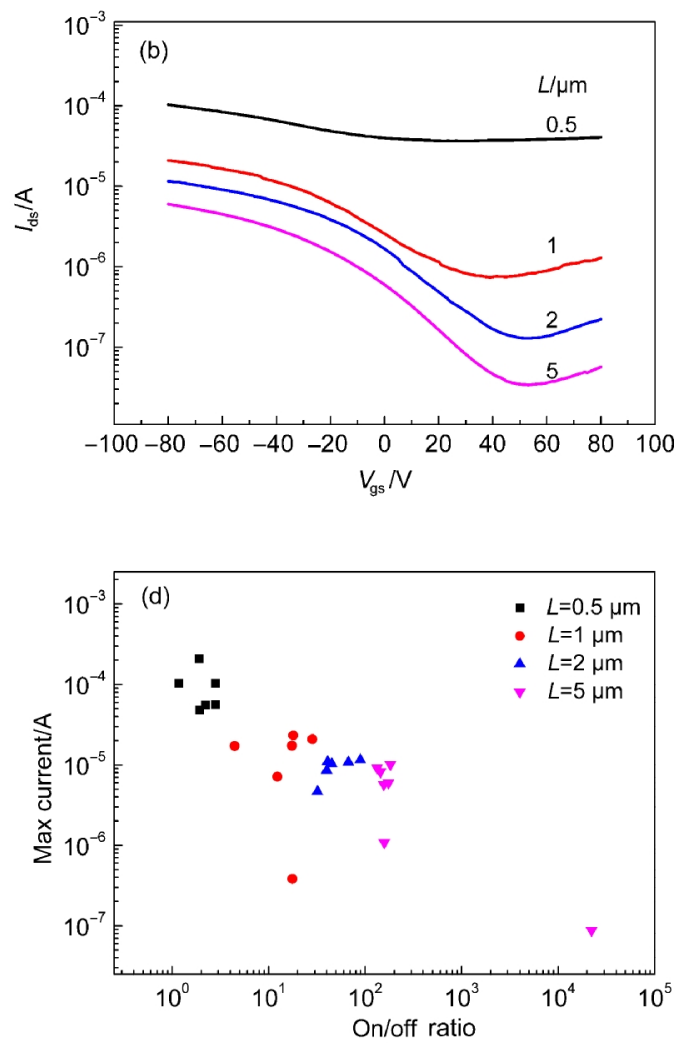

图 2 高纯半导体碳纳米管平行阵列的薄膜晶体管器件结构与电学特性

Fig.2 Device structure and electrical properties of thin-film transistors based on high purity semiconducting nanotube aligned arrays

(a) schematic of the CNT-TFT device with asymmetric contacts; (b) transfer characteristics of nanotube TFTs, with channel lengths of $0.5,1$, 2 , and $5 \mu \mathrm{m}$ under bias $1 \mathrm{~V}$; (c) current-voltage characteristic of TFT device with channel length $L=2 \mu \mathrm{m}$. $V_{\mathrm{gs}}$ changes from -40 to $80 \mathrm{~V}$ with a step of $40 \mathrm{~V}$ (gs: gate-source, ds: drain-source); (d) on-state current plotted against on/off ratio of thin film transistors for all devices studied 
$\mu \mathrm{m}$, 此时大部分碳管的两端可以直接搭接到源漏电 极上, 电流的输运机制以直接输运为主, 由于碳纳 米管的密度较高, 每微米超过 20 根, ${ }^{23}$ 因此同时也存 在渗透输运机制. 此时材料中的金属管的直接搭接 和渗透输运机制存在使得器件的电流基本不受底 栅调制, 开关比小于 10 . 当沟道长度为 $1 \mu \mathrm{m}$ 时, 器件 的开关比为 $10^{1}-10^{2}$. 随着沟道长度的进一步增加, 当沟道长度为 $2 \mu \mathrm{m}$ 时, 由于碳管平均长度只有 1 $\mu \mathrm{m}$ 左右, 器件中直接搭接到源漏电极的金属碳管几 乎不存在, 器件的开关比能够达到 $10^{2}$, 电流的输运 机制从直接输运为主变为渗透输运为主. 最后当沟 道长度为 $5 \mu \mathrm{m}$, 远大于碳管平均长度时, 开关比均 大于 $10^{2}$, 个别器件能大于 $10^{4}$, 此时载流子需要通过 多个碳管间的渗透输运才能从一个电极到达另一 个电极, 因此具有最大的开关比和最小的开态电 导.

图 2(c) 为沟道长度为 $2 \mu \mathrm{m}$ 的典型器件在不同 椓压下的输出特性. 此沟道长度器件的电流受到栅 压明显的调制, 开关比可以达到 2 个数量级. 在正负 偏压下的电流基本对称, 电阻随偏压的增大而减 小. 单根碳管在非对称接触 $\mathrm{Pd} / \mathrm{Sc}$ 结构会表现出的 典型二极管特性在薄膜体系中没有被观察到, 这主 要可能是由于薄膜体系的输运特性为渗透输运机 制, 并且与沟道中碳纳米管之间接触引入的肖特基 结有关, 沟道中碳管之间的多次搭接形成了一个较 为复杂的输运体系. 器件的电流电压特性 $\left(I_{\mathrm{ds}}-V_{\mathrm{ds}}\right)$ 由 非对称的金属与碳管接触形成的接触电阻和碳管 之间的接触特性共同决定. 图 2(d) 为同一批制作的 所有不同沟道长度器件的开态电流与开关比的关 系统计结果. 绝大多数相同沟道长度器件的开态电 流和开关比变化在一个较小的范围内. 实验结果显 示了相同沟道长度器件之间较高的均一性, 与此同 时不同沟道长度的器件开态电流和开关比的分布 有显著的差异. 长沟道的器件拥有较大的开关比和 较小的开态电流, 短沟道的器件开关比较小, 但开 态电流较大, 与图 2(b)转移曲线规律一致.

\section{2 器件的光电特性表征}

在前面电学特性表征的基础上, 我们对比了不 同沟道长度器件的红外光电响应. 图 3(a) 显示了无 光照、栅压处于关态条件下, 不同沟道长度的器件 的电流-电压 $(I-V)$ 特性. 沟道长度越长, 暗电流越 小. 图 3(b) 为沟道长度 $2 \mu \mathrm{m}$ 的典型器件在不同光强 条件下 (光照强度分别为 $2.3,1.15,0.58,0.23,0.023$
$\mathrm{kW} \cdot \mathrm{cm}^{-2}$, 激发波长 $785 \mathrm{~nm}$ ) 的 $I-V$ 特性. 入射功率 密度为 $2.3 \mathrm{~kW} \cdot \mathrm{cm}^{-2}$ 时, 短路光电流为 $6.3 \mathrm{nA}$. 光电 流随光强的减小而减小, 在光照强度下降至 0.023 $\mathrm{kW} \cdot \mathrm{cm}^{-2}$ 时, 器件仍存在一定的光电流响应. 由于碳 纳米管的光吸收具有偏振依赖关系, 在轴向有最强 的光吸收特性, 我们采用的激光偏振方向与碳纳米 管定向排列方向一致以获得高的光电响应效果. 图 3(c)不同颜色的点显示了不同沟道长度的器件短路 光电流随光照强度的变化趋势, 图中直线是拟合的 结果. 器件的短路光电流均随光照强度基本呈线性 变化, 长沟道的器件(大于 $1 \mu \mathrm{m}$ ) 的短路光电流较短 沟道的器件要小, 这是由于随着沟道长度变长, 载 流子被电极收集形成光电流的过程需要经过更多 碳管之间的输运导致的结果, 同时光生载流子也容 易在输运过程中产生复合而损耗掉. 对于 $0.5 \mu \mathrm{m}$ 短 沟道器件中, 由于存在金属管直接搭接源漏电极, 在光照情况下很难有效产生短路光电流和开路光 电压, 而沟道长度为 2 和 $5 \mu \mathrm{m}$ 的短路光电流值差异 较小.

为进一步了解碳纳米管薄膜体系中的有效的 光生载流子分离区域和分离机制, 我们采用激光扫 描光电流谱的方法对器件不同区域的光响应进行 了研究. 图 4(a)为高纯半导体碳纳米管阵列晶体管 对光照位置沿沟道方向的光电流响应. 测量采用沟 道长度为一个 $5 \mu \mathrm{m}$ 的典型器件, 激发波长为 633 $\mathrm{nm}$, 采用 50 倍的物镜进行聚焦, 激光的光斑直径约 为 $2 \mu \mathrm{m}$. 在关态栅压情况下, 通过移动样品台, 光斑 从器件漏电极一侧的沟道之外区域, 以 $1 \mu \mathrm{m}$ 步长沿 着器件沟道从漏电极往源电极进行扫描. 从图 4(a) 中可以看到, 器件在金属和碳纳米管接触处附近有 较大的正向光电流, 说明在该体系下, 光激发电子 空穴对的分离主要发生在金属和碳纳米管接触区 域. 而光照在器件沟道中间时, 由于没有有效的自 建场存在, 无法产生正向光电流, 因此沟道中间处 的反向电流可能是由碳管的局域缺陷引起的光电 流. 图 4(b) 显示了对应器件的能带示意图, 非对称接 触形成的能带弯曲是在两个电极接触区域产生净 光电流的主要条件. 在金属钯与碳纳米管接触区域 附近, 通过电荷转移, 形成空穴富集区域; 在金属铅 与碳纳米管接触区域附近, 形成电子富集区域, 所 以两个区域产生的光电流同向. 当激光照射在碳管 与 $\mathrm{Pd}$ 金属接触处的时候, 由于碳管的能带向上弯曲 且 $P d$ 金属可以与碳纳米管的价带形成良好的欧姆 

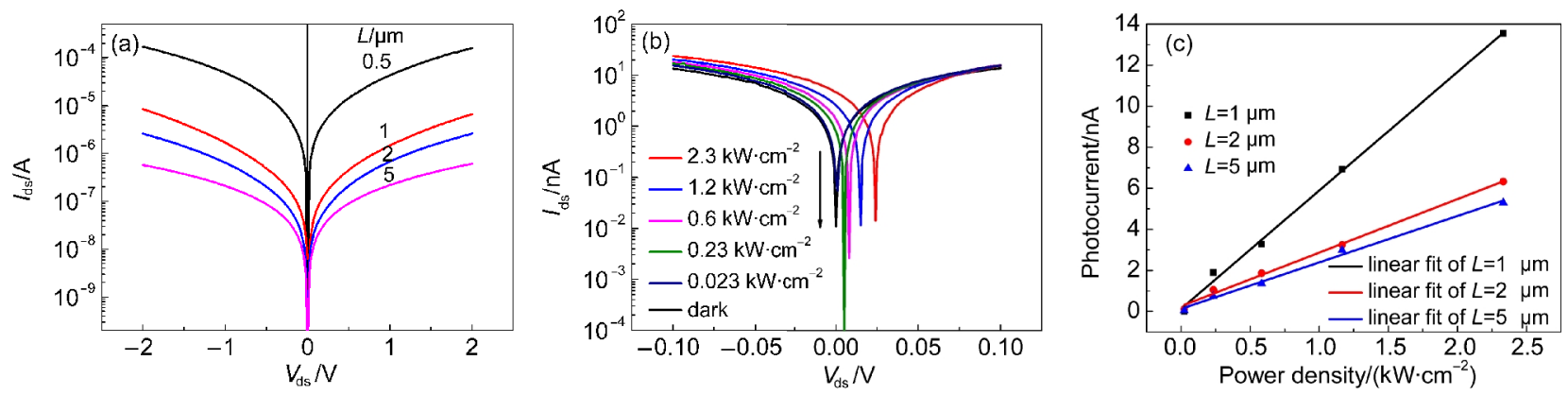

图 3 高纯半导体碳纳米管阵列薄膜晶体管的光电特性表征

Fig.3 Photoelectric properties of thin-film transistors based on high purity semiconducting nanotube arrays

(a) current-voltage $(I-V)$ characteristic of typical CNT-TFTs with channel lengths of $0.5,1,2$, and $5 \mu \mathrm{m}$; (b) current-voltage characteristics in a logarithmic scale of a typical CNT-TFT with channel length of $2 \mu \mathrm{m}$ with different power densities and the laser wavelength of $785 \mathrm{~nm}$;

(c) experimental data (point) and fit results (line) for short circuit current as a function of power density, for TFT

devices with channel lengths of 1,2 , and $5 \mu \mathrm{m}$

接触, 光激发的空穴很容易被 Pd 电极收集, 产生净 的光电流. 当激光照射在碳管与 $\mathrm{Sc}$ 金属接触处时, 由于碳管的能带向上弯曲且 Sc 金属可以与碳纳米 管的导带形成良好的欧姆接触. 光激发的电子很快 被 Sc 电极收集, 产生与光照在 Pd 端同向的光电流. 而在沟道中间, 由于远离接触区域, 没有有效分离 电子空穴的自建电场, 光电流迅速变小, 光激发的
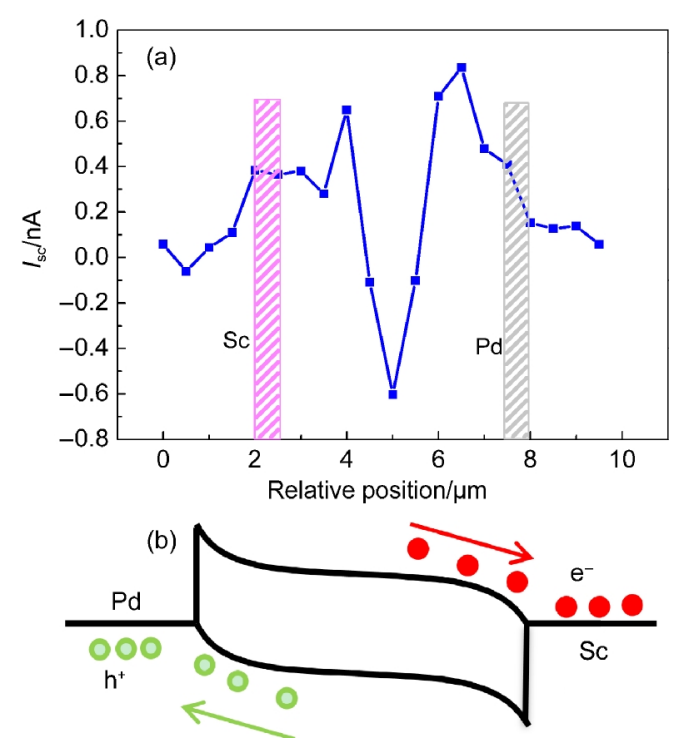

图 4 高纯半导体碳纳米管阵列晶体管沿沟道方向的 光电流响应 (激光波长 $\lambda=633 \mathbf{n m}$ )

Fig.4 Spatial photocurrent response along the channel of high purity semiconducting CNT-TFTs device with scanning laser wavelength of $633 \mathbf{~ n m}$

(a) short circuit current $\left(I_{\mathrm{sc}}\right)$ as a function of position being illuminated when gate bias $\left(V_{\mathrm{gs}}\right)$ is $20 \mathrm{~V}$; (b) depicted energy band diagrams of the asymmetrically contacted CNT-TFT. The electron-hole pairs can be separated and collected efficiently at the contact area between metal electrode and carbon nanotube.
载流子无法有效通过碳管之间输运到达源漏电极.

\section{4 结 论}

采用溶液蒸发自组装法(EDSA)可控地大面积 定向排列了 $99 \%$ 高纯半导体单壁碳纳米管阵列薄 膜. 利用排列的碳纳米管阵列, 采用无掺杂的非对 称接触电极方法制备了薄膜晶体管器件, 对它的电 学特性、光电特性进行了测量和研究, 并对相关的 物理机制进行了分析. 在高纯碳管阵列薄膜器件 中, 沟道长度对器件的电学、光电响应特性存在较 大影响. 器件的沟道长度超过碳纳米管的平均长度 会使沟道中管与管之间的结数量增加, 使器件由直 接输运占主导转变为渗透输运占主导, 同时器件的 电导和短路光电流下降, 开关比增加. 扫描光电流 结果也显示出光电流主要产生在碳管与金属 $(\mathrm{Pd}$ 和 $\mathrm{Sc}$ )接触区域附近, 同时非对称的接触电极可以高效 地收集光生载流子. 相关结果为高纯碳管薄膜在光 探测方面的应用提供了参考依据.

\section{References}

(1) Iijima, S. Nature 1991, 354, 56. doi: 10.1038/354056a0

(2) Perebeinos, V.; Tersoff, J.; Avouris, P. Nano Lett. 2006, 6, 205. doi: $10.1021 / \mathrm{n} 1052044 \mathrm{~h}$

(3) Purewal, M. S.; Hong, B. H.; Ravi, A.; Chandra, B.; Hone, J.; Kim, P. Phys. Rev. Lett. 2007, 98, 186808. doi: 10.1103/ PhysRevLett.98.186808

(4) Cao, Q.; Kim, H. S.; Pimparkar, N.; Kulkarni, J. P.; Wang, C.; Shim, M.; Roy, K.; Alam, M. A.; Rogers, J. A. Nature 2008, 454, 495. doi: 10.1038/nature07110

(5) Liang, X. L.; Wang, S.; Wei, X. L.; Ding, L.; Zhu, Y. Z.; Zhang, Z. Y.; Chen, Q.; Li, Y.; Zhang, J.; Peng, L. M. Adv. Mater. 2009, 
21, 1339. doi: 10.1002/adma.v21:13

(6) Zhu, Y. Z.; Wang, S.; Wei, X. L.; Ding, L.; Zhang, Z. Y.; Liang, X. L.; Chen, Q.; Peng, L. M. Acta Phys. -Chim. Sin. 2008, 24, 2122. [朱玉振, 王 胜, 魏贤龙, 丁 力, 张志勇, 梁学磊, 陈 清, 彭练矛, 物理化学学报, 2008, 24, 2122] doi: 10.3866/PKU. WHXB20081131

(7) Bachilo, S. M.; Strano, M. S.; Kittrell, C.; Hauge, R. H.; Smalley, R. E.; Weisman, R. B. Science 2002, 298, 2361. doi: $10.1126 /$ science. 1078727

(8) Itkis, M. E.; Borondics, F.; Yu, A.; Haddon, R. C. Science 2006, 312, 413. doi: 10.1126/science. 1125695

(9) Yang, L. J.; Wang, S.; Zeng, Q. S.; Zhang, Z. Y.; Li, Y.; Zhou, W. W.; Liu, J.; Peng, L. M. ACS Appl. Mater. Inter. 2012, 4, 1154. doi: $10.1021 / \mathrm{am} 201778 \mathrm{x}$

(10) Tans, S. J.; Verschueren, A. R. M.; Dekker, C. Nature 1998, 393, 49. doi: $10.1038 / 29954$

(11) Javey, A.; Guo, J.; Wang, Q.; Lundstrom, M.; Dai, H. J. Nature 2003, 424, 654. doi: 10.1038/nature01797

(12) Zhang, Z.; Liang, X.; Wang, S.; Yao, K.; Hu, Y.; Zhu, Y.; Chen, Q.; Zhou, W.; Li, Y.; Yao, Y.; Zhang, J.; Peng, L. M. Nano Lett. 2007, 7, 3603. doi: 10.1021/n10717107

(13) Ding, L.; Wang, S.; Zhang, Z. Y.; Zeng, Q. S.; Wang, Z. X.; Pei, T.; Yang, L. J.; Liang, X. L.; Shen, J.; Chen, Q.; Cui, R. L.; Li, Y.; Peng, L. M. Nano Lett. 2009, 9, 4209. doi: 10.1021/ n19024243

(14) Wang, S.; Zhang, Z. Y.; Ding, L.; Liang, X. L.; Sun, J.; Xu, H. L.; Chen, Q.; Cui, R. L.; Li, Y.; Peng, L. M. Adv. Mater. 2008, 20, 3258. doi: 10.1002/adma.v20:17

(15) Yang, L.; Wang, S.; Zeng, Q. S.; Zhang, Z. Y.; Pei, T.; Li, Y.;
Peng, L. M. Nature Photon. 2011, 5, 672. doi: 10.1038/ nphoton.2011.250

(16) Yang, L.; Wang, S.; Zeng, Q. S.; Zhang, Z. Y.; Peng, L. M. Small 2013, 9, 1225. doi: 10.1002/smll.201203151

(17) Snow, E. S.; Novak, J. P.; Campbell, P. M.; Park, D. Appl. Phys. Lett. 2003, 82, 2145. doi: 10.1063/1.1564291

(18) Zhang, J.; Wang, C.; Fu, Y.; Che, Y.; Zhou, C. W. ACS Nano 2011, 5, 3284. doi: 10.1021/nn2004298

(19) Wang, C.; Zhang, J.; Zhou, C. W. ACS Nano 2010, 4, 7123. doi: $10.1021 / \mathrm{nn} 1021378$

(20) Wei, J. Q.; Jia, Y.; Shu, Q. K.; Gu, Z. Y.; Wang, K. L.; Zhuang, D. M.; Zhang, G.; Wang, Z. C.; Luo, J. B.; Cao, A. Y.; Wu, D. H. Nano Lett. 2007, 7, 2317. doi: 10.1021/n1070961c

(21) Wang, C.; Zhang, J.; Ryu, K.; Badmaev, A.; Arco, L. G.; Zhou, C. W. Nano Lett. 2009, 9, 4285. doi: 10.1021/n1902522f

(22) Shastry, T, A.; Seo, J.; Lopez, J.; Arnold, H.; Kelter J.; Sangwan, V.; Lauhon, L.; Marks, T.; Hersam, M. C. Small 2013, 9, 45. doi: 10.1002/smll.v9.1

(23) Engel, M.; Small, J. P.; Steiner, M.; Freitag, M.; Green, A. A.; Hersam, M. C.; Avouris, P. ACS Nano 2008, 2, 2445. doi: $10.1021 / \mathrm{nn} 800708 \mathrm{w}$

(24) Kinoshita, M.; Steiner, M.; Engel, M.; Small, J. P.; Green, A. A.; Hersam, M. C.; Krupke, R.; Mendez, E. E.; Avouris, P. Opt. Express 2010, 18, 25738. doi: 10.1364/OE.18.025738

(25) Dresselhaus, M. S.; Dresselhaus, G.; Saito, R.; Jorio, A. Phys. Rep. 2005, 409, 47. doi: 10.1016/j.physrep.2004.10.006

(26) Cao, Q.; Han, S.; Tulevski, G. S.; Zhu, Y.; Lu, D. D.; Haensch, W. Nature Nanotech. 2013, 8, 180. doi: 10.1038/nnano.2012.257 